\title{
STATIONARY DENSE OPERATORS IN SEQUENTIALLY COMPLETE LOCALLY CONVEX SPACES
}

\author{
DANIEL VELINOV, MARKO KOSTIĆ, AND STEVAN PILIPOVIĆ
}

\begin{abstract}
The purpose of this paper is to investigate the stationary dense operators and their connection to distribution semigroups and abstract Cauchy problem in sequentially complete spaces.
\end{abstract}

\section{Introduction AND Preliminaries}

In [15] and [12] are given definitions of a distribution semigroups with densely and non densely defined generators. Stationary dense operators in a Banach space $E$ and their connections to densely defined distribution semigroups are introduced and analyzed in 11. Our main aim in this paper is to consider such operators when $E$ is sequentially complete locally convex space.

When we are dealing with a semigroup in a locally convex space, in order to have the existence of the resolvent of an infinitesimal generator $A$, we suppose that the semigroup is equicontinuous. Moreover for $A$, we are led to study the behavior of $A_{\infty}$ in $D_{\infty}(A)$. If $A$ is stationary dense, the information lost in passing from $A$ in $E$ to $A_{\infty}$ in $D_{\infty}(A)$, can be retrieved. Let us emphasize that the interest for the stationarity of $A$ comes out from the solvability of the Cauchy problem $u^{\prime}=A u$, $u(0)=u_{0}$, for every $u \in D\left(A^{n}\right)$ for some $n$. This is noted in Proposition 2.3 In Theorem 2.12 we have a partial answer to the converse question: Whether the solvability for every $x \in D\left(A^{n}\right)$ implies $n$-stationarity. Also, we extend the results of [2, 11], 18 and give new examples of stationary dense operators on sequentially complete locally convex spaces.

Here we denote with $E$ a sequentially complete locally convex space with system of seminorms $\circledast$. For a closed linear operator $A$ we define $D_{\infty}(A)=\bigcap_{n=1}^{\infty} D\left(A^{n} x\right)$, for $x \in E$. Then the space $D_{\infty}(A)$ is Fréchet space as a projective limit of Fréchet spaces with seminorms $p_{n}(x)=\sup _{p \in \circledast} \sum_{k=0}^{n} p\left(A^{k} x\right)$, for for all $q \in \circledast$ and $x \in D_{\infty}(A) \subseteq$ $E, n \in \mathbb{N}$.

The distribution semigroups are defined by ([15):

A distribution $G$ is a distribution semigroup (shortly $D S G$ ) if following conditions are satisfied:

(i) $G \in \mathcal{D}_{+}^{\prime}(L(E ; E)), \quad G=0, \quad$ for $\quad t<0$;

(ii) $G(\varphi * \psi)=G(\varphi) G(\psi)$ for all $\varphi, \psi \in \mathcal{D}_{0}$, where $\mathcal{D}_{0}$ is the space of all $\varphi \in \mathcal{D}$ such that $\varphi(t)=0$ for $t<0$;

2010 Mathematics Subject Classification. 35R11, 45D05, 45N05, 47D99.

Key words and phrases. stationary dense operators, distribution semigroups, ultradistribution semigroups, well-posedness, locally convex spaces.

* This research is partially supported by grant 174024 of Ministry of Science and Technological Development, Republic of Serbia. 
(iii) Let $\varphi \in \mathcal{D}_{0}, x \in E$ and $y=G(\varphi) x$. The distribution $G y$ is equal to a continuous function $u$ on $[0,+\infty)$ and $u(t)=0$ for $t<0$ with range in $E$ and $u(0)=y$;

(iv) The range of the all elements $G(\varphi) x$, where $\varphi \in \mathcal{D}_{0}, x \in E$ is dense in $E$;

(v) If for all $\varphi \in \mathcal{D}_{0}, G(\varphi) x=0, x \in E$ then $x=0$.

In further consideration will be used the last definition of distribution semigroups, although often are considered semigroups which are not densely defined (cf. [12]). In order to give the definition of exponential distribution semigroups we give the definition of the spaces through tempered distributions. Let $a \geq 0$. Then

$$
\mathcal{S E}_{a}(\mathbb{R}):=\left\{\phi \in C^{\infty}(\mathbb{R}): e^{a t} \phi \in \mathcal{S}(\mathbb{R})\right\} .
$$

Define the convergence in this space by

$$
\phi_{n} \rightarrow 0 \text { in } \mathcal{S E}_{a}(\mathbb{R}) \text { iff } e^{a \cdot} \phi_{n} \rightarrow 0 \text { in } \mathcal{S}(\mathbb{R}) .
$$

Denote by $\mathcal{S E}_{a}^{\prime}(\mathbb{R}, E)$ the space $L\left(\mathcal{S E}_{a}(\mathbb{R}), E\right)$ which is formed from all continuous linear mappings from $\mathcal{S E}_{a}(\mathbb{R})$ into $E$ equipped with the strong topology.

It holds,

$$
F \in \mathcal{S E}_{a}^{\prime}(\mathbb{R}, E) \text { if and only if } e^{-a \cdot} F \in \mathcal{S}^{\prime}(\mathbb{R}, E) .
$$

In sequel will be given the structure of the space $\mathcal{S E}_{a}^{\prime}(\mathbb{R}, E)$ used in definition of exponential distribution semigroups.

Proposition 1.1. Let $T \in \mathcal{S E}_{a}^{\prime}(\mathbb{R}, E)$. Then there exists an polynomial $P$ and a function $g \in C(\mathbb{R}, E)$ with the property that there exist $k>0$ and $C>0$, such that

$$
e^{-a x}|| g(x) \| \leq C|x|^{k}, x \in \mathbb{R} \text { and } T=P(d / d t) g .
$$

The proof of this proposition is similar like in ultradistribution case, even more simple (see [9, 10, for ultradistribution and hyperfunction case, when $E$ is a Banach space), hence will be omitted.

A distribution semigroup $G$ is an exponential distribution semigroup, ( $E$-distribution semigroup, $(E D S G)$ shortly), if in addition to $(i)-(v), G$ fulfills:

$$
\text { (vi) } \left.(\exists a \geq 0) G \in \mathcal{S} E_{a}^{\prime}(\mathbb{R}, L(E))\right) .
$$

The generator $A$ of a distribution semigroup $G$ is defined as

$$
A=\left\{(x, y) \in E^{2}: \forall \varphi \in \mathcal{D}, G\left(-\varphi^{\prime}\right) x=G(\varphi) y\right\} .
$$

Also we want to refer to [15] and [12] for structural theorems for distribution semigroups and exponential semigroups (for ultradistribution case see [9, Theorem 2.7]). An $C_{0}$-semigroup $(T(t))_{t \geq 0}$ in sequentially complete locally convex space $E$ is an equicontinuous semigroup if the set $\{T(t): t \geq 0\}$ is an equicontinuous set in $E$. Moreover, we say that an $C_{0}$-semigroup is quasi-equicontinuous if there is an $a \geq 0$ such that $\left\{e^{-a t} T(t): t \geq 0\right\}$ is an equicontinuous set.

Let $D$ and $E$ are sequentially complete locally convex spaces and let $P \in$ $\mathcal{D}_{0}^{\prime}(L(D, E))$. Then $G \in \mathcal{D}_{0}^{\prime}(L(E, D))$ is said to be a distribution fundamental solution for $P=\delta^{\prime} \otimes I-\delta \otimes A$ when $P * G=\delta \otimes I_{E}$ and $G * P=\delta \otimes I_{D}$. Let $E$ be a sequentially complete locally convex space. In [16], is given structural theorem for $E$-valued distributions and there is an analogue in the case of $E$-valued tempered distributions which can be proved similar like in ultradistribution case. Next theorem is a structural theorem for distribution semigroups. Actually, a version of this theorem is given in [9] for ultradistribution semigroups when $E$ is a Banach space. The proof is similar. 
Theorem 1.2. $\quad$ (a) A generates a (DSG) G.

(a)' A generates a (EDSG) G.

(b) A generates a $(D S G) G$ such that for every $a>0, G$ is of the form $G=P_{L}^{a}(-i d / d t) S_{K}^{a}$ on $\mathcal{D}((-\infty, a))$ where $S_{K}^{a}:(-\infty, a) \rightarrow L(E,[D(A)])$ is continuous, $S_{K}^{a}(t)=0, t \leq 0$.

(b)' A generates a (EDSG) $G$ so that $G$ is of the form $G=P_{L}(-i d / d t) S_{K}$ on $\mathcal{S E}_{a}(\mathbb{R})$, where $S_{K}: \mathbb{R} \rightarrow L(E,[D(A)])$ is continuous, $S_{K}(t)=0, t \leq 0$ and $e^{-a t}|| S_{k}(t) \| \leq A|t|^{k}$, for some $k>0$ and $A>0$.

(c) For every a $>0, A$ is the generator of a local non-degenerate $K_{a}$-convoluted semigroup $\left(S_{K_{a}}^{a}(t)\right)_{t \in[0, a)}$, where $K_{a}=\mathcal{L}^{-1}\left(\frac{1}{P_{L}^{a}(-i \lambda)}\right)$ and $P_{L}^{a}$ is a differential operator such that for $0<a<b$ the restriction of $P_{L}^{b} S_{K}^{b}$, on $\mathcal{D}((-\infty, a))$ is equal to $P_{L}^{a} S_{K}^{a}$.

(c)' $A$ is the generator of a global, exponentially bounded non-degenerate $K$ convoluted semigroup $\left(S_{K}(t)\right)_{t \geq 0}$, where $K=\mathcal{L}^{-1}\left(\frac{1}{P_{L}(-i \lambda)}\right)$.

(d) There exists an distribution fundamental solution for $A$, denoted by $G$, with the property $\mathcal{N}(G)=\{0\}$.

(d)' There exists an exponential distribution fundamental solution $G$ for $A$, with the property $\mathcal{N}(G)=\{0\}$.

(e) $\rho(A) \supset \Omega_{\alpha, \beta}=\{\xi+i \eta: \alpha \log (1+|\eta|)+\beta\}$ and

$$
\|R(\lambda: A)\| \leq C(1+|\lambda|)^{k}, \quad \lambda \in \Omega_{\alpha, \beta},
$$

for some $k>0$ and $C>0$.

(e)' $\rho(A) \supset\{\lambda \in \mathbb{C}: \operatorname{Re} \lambda>a\}$ and

$$
\|R(\lambda: A)\| \leq C(1+|\lambda|)^{k}, \operatorname{Re} \lambda>a,
$$

for some $a, k>0$ and $C>0$.

Then, $(a) \Leftrightarrow(d) ;(a)^{\prime} \Leftrightarrow(d)^{\prime} ;(c) \Rightarrow(d) ;(c)^{\prime} \Rightarrow(d)^{\prime} ;(d) \Leftrightarrow(e) ;(d)^{\prime} \Leftrightarrow(e)^{\prime}$; $(a)^{\prime} \Rightarrow(c)^{\prime}$.

Note that the case of exponential distribution semigroup case is given parallel and it is denoted with '. The proof of this theorem is similar (even simpler) with [9, Theorem 2.7], so we omit it.

\section{Stationary DENSE operators in LOCALly CONVEX SPACES}

Following P. C. Kunstmann [11, we introduce the notion of a stationary dense operator in a sequentially complete locally convex space as follows.

Definition 2.1. A closed linear operator $A$ is said to be stationary dense iff

$$
n(A):=\inf \left\{k \in \mathbb{N}_{0}: D\left(A^{m}\right) \subseteq \overline{D\left(A^{m+1}\right)} \text { for all } m \geq k\right\}<\infty .
$$

Example 2.2. Let $E=L^{p}(\mathbb{R}), 1 \leq \leq \infty$. We consider the multiplication operator with maximal domain in $E$ :

$$
A f(x)=\left(x+i x^{2}\right) f(x),
$$

$x \in \mathbb{R}, f \in E$. It is clear that $A$ is dense and stationary dense if $1 \leq p<\infty$. If $p=\infty$, then $A$ is not stationary dense. Indeed, the function $g(x)=\frac{1}{x^{2 n}+1}$ belongs to $D\left(A^{n}\right) \backslash \overline{D\left({ }^{n+1}\right)}$, for $n \in \mathbb{N}$. 
The abstract Cauchy problem

$$
\left(A C P_{1}\right):\left\{\begin{array}{l}
u \in C([0, \tau):[D(A)]) \cap C^{1}([0, \tau): E), \\
u^{\prime}(t)=A u(t), t \in[0, \tau), \\
u(0)=x
\end{array}\right.
$$

where $A$ is a closed linear operator on $E$ and $0<\tau \leq \infty$, has been analyzed in a great number of research papers and monographs (see e.g. [1], [3]-6], [7], [8][9], 11]-15, [17] and [18]). By a mild solution of problem $\left(A C P_{1}\right)$ we mean any continuous function $t \mapsto u(t ; x), t \in[0, \tau)$ such that $A \int_{0}^{t} u(s ; x) d s=u(t ; x)-x$, $t \in[0, \tau)$.

Proposition 2.3. Let $0<\tau \leq \infty$ and $n \in \mathbb{N}_{0}$.

(i) Suppose that the abstract Cauchy problem $\left(A C P_{1}\right)$ has a unique mild solution $u(t ; x)$ for all $x \in D\left(A^{n}\right)$. Then $A$ is stationary dense and $n(A) \leq n$.

(ii) Suppose that $\left(S_{n}(t)\right)_{t \in[0, \tau)}$ is a locally equicontinuous $n$-times integrated semigroup generated by $A$. Then $A$ is stationary dense and $n(A) \leq n$.

Proof. One has to use the arguments given in that of [11, Lemma 1.7] (cf. also [11, Remark 1.2(i)]) and the fact that for any locally equicontinuous $n$-times integrated semigroup $\left(S_{n}(t)\right)_{t \in[0, \tau)}$ generated by $A$ the abstract Cauchy problem $\left(A C P_{1}\right)$ has a unique mild solution for all $x \in D\left(A^{n}\right)$, given by $u(t ; x)=S_{n}(t) A^{n} x+\sum_{j=0}^{n-1} \frac{t^{j}}{j !} A^{j} x$, $t \in[0, \tau)$.

Lemma 2.4. Let $A$ be a closed operator in sequentially complete locally convex space and let $\left(\lambda_{n}\right) \in \rho(A)$ be a sequence such that $\lim _{n \rightarrow \infty}\left|\lambda_{n}\right|=\infty$ and there exist $C>0$ and $k \geq-1$ such that for every $p \in \circledast$, there exists $q \in \circledast$, such that $p\left(R\left(\lambda_{n}: A\right) x\right) \leq C\left|\lambda_{n}\right|^{k} q(x)$ for all $x \in E$ and $n \in \mathbb{N}$. Then $A$ is stationary dense with $n(A) \leq k+2$.

Proof. Let $x \in D\left(A^{k+1}\right)$. Then for every $p \in \circledast$, there exists $q \in \circledast$, such that $p\left(\lambda_{n} R\left(\lambda_{n}: A\right) x\right) \leq\left\|R\left(\lambda_{n}: A\right)\right\| q(A x)$. Now for $x \in D\left(A^{k+2}\right)$, it follows $\lambda_{n} R\left(\lambda_{n}\right.$ : A) $x \in D\left(A^{k+3}\right)$ for all $\mathbb{N}$. Furthermore

$$
p\left(\lambda_{n} R\left(\lambda_{n}: A\right) x-x\right)=p\left(R\left(\lambda_{n}: A\right) A x\right) \leq \frac{C^{\prime}}{\left|\lambda_{n}\right|} q(A x) .
$$

Hence,

$$
x=\lim _{n \longrightarrow \infty} \lambda_{n} R\left(\lambda_{n}: A\right) x
$$

and $x$ belongs to the closure of $D\left(A^{k+3}\right)$, which means that $A$ is stationary dense and $n(A) \leq k+2$.

Example 2.5. The basic idea for this example is from [1]. Let $E$ be a sequentially complete locally convex space and $A$ is a closed linear operator in $E$ which is not stationary dense and there exist $C, k>0$ (for all $k>0$ there exist $C_{k}>0$ ) such that

$$
p(R(\lambda: A) x) \leq C e^{M(k|\lambda|)} p(x), \Re \lambda \geq 0 .
$$

We construct inductively an increasing sequence $\left(M_{n}\right)_{n \in \mathbb{N}_{0}}, M_{0}=1$, and satisfying (M.1), (M.3)' and for all $\lambda \geq 0$

$$
\sum_{p=0}^{\infty} \frac{\lambda^{p}}{M_{p}} \leq C_{k} e^{M(k|\lambda|)}
$$


The space $E_{M_{n}}$ is defined as

$$
E_{M_{n}}=\left\{f \in \mathcal{C}^{\infty}([0,1], E):\|f\|_{M_{n}, h}=\sup _{n} \frac{p\left(f^{(n)}\right)}{M_{n} h^{n}}<\infty, \forall n \in \circledast\right\} .
$$

Let $A=-\frac{d}{d s}$, with domain $D(A)=\left\{f \in E_{M_{n}}: f^{\prime} \in E_{M_{n}}, f(0)=0\right\}$. Let $g(t)=R(\lambda: A) f(t)=e^{-\lambda t} \int_{0}^{t} e^{\lambda s} f(s) d s=\int_{0}^{t} e^{-\lambda(t-s)} f(s) d s$. Then $g(0)=0, g$ is smooth function and $\lambda g+g^{\prime}=f$. By

$$
g^{(n)}=f^{(n-1)}+\sum_{k=1}^{n-1}(-\lambda)^{k} f^{(n-1-k)}+(-\lambda)^{n} g
$$

and $p(R(\lambda: A) f) \leq p(f)$ we have

$$
\frac{p\left(g^{(n)}\right)}{M_{n} h^{n}} \leq \frac{p\left(f^{(n-1)}\right)}{M_{n-1} h^{n-1}}+\sum_{k=1}^{n-1} \frac{|\lambda|^{k}}{M_{k} M_{n-1-k} h^{n-1-k}} p\left(f^{(n-1-k)}\right)+\frac{|\lambda|^{n}}{M_{n} h^{n}} p(f) .
$$

Hence,

$$
\|g\|_{M_{n}, h} \leq \sum_{k=0}^{\infty} \frac{|\lambda|^{k}}{M_{k}}\|f\|_{M_{n}, h} \leq C e^{M(k|\lambda|)}\|f\|_{M_{n}, h}
$$

which means that $g \in E_{M_{n}}$. Moreover, $g \in D(A)$ and $\|R(\lambda: A)\| \leq C e^{M(k|\lambda|)}$ and $A$ is a generator of an ultradistribution semigroup.

The domain of the operator $A^{k}$ is $D\left(A^{k}\right)=\left\{f \in E_{M_{n}}: \forall j \in\{0,1,2, \ldots, k-\right.$ $\left.1\}, f^{(j-1)} \in E_{M_{n}}, f^{(j)}(0)=0\right\}$ and $\overline{D\left(A^{k}\right)} \subset\left\{f \in E_{M_{n}} . \forall j \in\{0,1,2, \ldots, k-\right.$ $\left.1\}, f^{(j)}(0)=0\right\}, k \in \mathbf{N}_{0}$. We consider the function $f(t)=\frac{t^{k}}{k !}$. Then $f \in D\left(A^{k}\right)$, but not in $\overline{D\left(A^{k+1}\right)}$, since $f^{(k)}(0)=1$. Hence $A$ is not stationary dense operator.

We say that the operator $A$ satisfy the condition $(E Q)$ if:

$A_{\infty}$ is a generator of an equicontinuous semigroup $T_{\infty}(t)$ in $D_{\infty}(A)$, i.e. for every $p \in \circledast$ there exists $q \in \circledast$ and $C$ such that

$$
p\left(T_{\infty}(t) x\right) \leq C q(x),
$$

for every $x \in D_{\infty}(A)$.

Using the results given in [11, [18, Theorem 4.1] we can state similar results in our setting ( $\mathrm{E}$ is sequentially complete locally convex space). Assume that $A$ is stationary dense, satisfies $(E Q), n=n(A)$ and $F$ is the closure of $D\left(A^{n}\right)$ in $E$.

Lemma 2.6. $\quad$ a) $A_{F}$ is densely defined in $F$, where $A_{F}$ means the restriction of the operator $A$ on $F$;

b) $\rho(A ; L(E))=\rho\left(A_{F} ; L(F)\right)$ for all $\lambda \in \rho(A)$. Additionally for all $x \in E$ and $p \in \circledast$, there exist $n \in \mathbb{N}, C>0$ such that

$$
p(R(\lambda: A) x) \leq C(1+|\lambda|)^{n}\left(p\left(R\left(\lambda: A_{F}\right) x\right)+1\right) ;
$$

c) The Fréchet spaces $D_{\infty}(A)$ and $D_{\infty}\left(A_{F}\right)$ coincide topologically and $A_{\infty}=$ $\left(A_{F}\right)_{\infty}$;

d) $n(A)=\inf \left\{k \in \mathbf{N}_{0}: \overline{D\left(A^{k}\right)} \subset D_{\infty}(A)\right\}$.

Proof. $\quad$ a) It is obvious since $D\left(A^{n}\right)$ is dense in $F$ and $D\left(A^{n}\right) \subset D\left(A_{F}\right)$. 
b) Since $D\left(A^{n}\right) \subset D(A), F$ is invariant under resolvent of $A$, we obtain $\rho(A ; L(E)) \subset \rho\left(A_{F} ; L(F)\right)$. Now, let $\lambda \in \rho\left(A_{F} ; L(F)\right)$. Then $\lambda-A$ is injective, so $R(\lambda: A)$ is an extension of $R\left(\lambda: A_{F}\right)$. Let $\mu \in \rho(A)$. By $R(\lambda: A)=(\mu-A)^{n} R(\lambda: A) R(\mu: A)^{n}$, we obtain that $\lambda \in \rho(A: L(E))$.

It holds, for $x \in E$ and all $p \in \circledast$,

$$
p(R(\lambda: A) x) \leq p_{n}\left(R(\mu: A)^{n} x\right) p_{n}\left(R\left(\lambda: A_{F}\right) x\right) p\left(R(\mu: A)^{n} x\right)
$$

where $p_{n}(x)=\sup _{p \in \circledast} \sum_{i=1}^{n} p\left(A^{i} x\right)$. Note that $\left(D\left(A^{n}\right), p_{n}\right)$ is a Banach space. For $x \in D\left(A^{n}\right)$ we have

$$
\begin{gathered}
p_{n}\left(R\left(\lambda: A_{F}\right) x\right)=\sup _{p \in \circledast} \sum_{i=0}^{n} p\left(A^{i} R\left(\lambda: A_{F}\right) x\right)= \\
=\sup _{p \in \circledast} \sum_{i=0}^{n} p\left(\left(\lambda^{k}+A^{k}-\lambda^{k}\right) R\left(\lambda: A_{F}\right) x\right) \leq \\
\leq \sup _{p \in \circledast} \sum_{i=0}^{n}\left(|\lambda|^{k} p\left(R\left(\lambda: A_{F}\right) x\right)+\sum_{j=0}^{i-1}|\lambda|^{j} p\left(A^{i-1-j} x\right)\right) .
\end{gathered}
$$

The last one inequality, together with the previous one gives the statement of the lemma.

c) It holds $D\left(A^{n+k}\right) \hookrightarrow D\left(\left(A_{F}\right)^{k}\right) \hookrightarrow D\left(A^{k}\right)$, which one holds for all $k \in \mathbb{N}_{0}$. Then

$$
\bigcap_{k=1}^{\infty} D\left(A^{n+k}\right) \hookrightarrow \bigcap_{k=1}^{\infty} D\left(A_{F}^{k}\right) \hookrightarrow \bigcap_{k=1}^{\infty} D\left(A^{k}\right),
$$

which gives that $D_{\infty}(A)$ and $D_{\infty}\left(A_{F}\right)$ coincide topologically and $A_{\infty}=$ $\left(A_{F}\right)_{\infty}$.

d) From the previous lemma, $D_{\infty}(A)=D_{\infty}\left(A_{F}\right)$ and $D\left(A^{n+1}\right) \subset D\left(A_{F}\right)$, we obtain the conclusion of the lemma.

Theorem 2.7. Let $A$ be a stationary dense operator in $E$ with non-empty resolvent. Then $\rho(A ; L(E))=\rho\left(A_{\infty} ; L\left(D_{\infty}(A)\right)\right)$.

Proof. By Lemma 2.6 a) we have that $A_{F}$ is dense in $F$ and by b) from the same lemma it has non-empty resolvent. Then using the proof of [11, Theorem 2.3] and Lemma 2.6 c), we obtain that $\rho(A ; L(E))=\rho\left(A_{\infty} ; L\left(D_{\infty}(A)\right)\right)$.

Next example show us if $A$ is not stationary dense operator in $E$, then the conclusion of Theorem 2.7 does not hold.

Example 2.8. Let we define the space $S_{j}$ as

$$
S_{j}=\left\{\varphi \in \mathcal{C}^{\infty}(\mathbb{R}): p_{j}(x)=\sup _{\alpha+\beta \leq j}\left\|x^{\beta} D^{\alpha} \varphi(x)\right\|_{L^{2}(\mathbb{R})}<\infty\right\} .
$$

Then the test space for tempered distributions $S(\mathbb{R})$ can be defined as $\mathcal{S}(\mathbb{R})=$ $\lim _{j \rightarrow \infty} \operatorname{proj} S_{j}$. Let $E=\mathcal{S}(\mathbb{R}),(E$ is a Fréchet space as a projective limit of Banach spaces, so $E$ is a sequentially complete locally convex space). Define $A=-\frac{d}{d t}$ on $E$ with domain $D(A)=\{f \in E: f(0)=0\}$. The operator $A$ is not stationary dense 
on $E$. Note that $D_{\infty}(A)=\{0\}$ and $\rho\left(A_{\infty}\right)=\{\lambda \in \mathbb{C}: \lambda \neq 0\}$. For $f \in E, \lambda \in \mathbb{C}$, and $\Re \lambda>s$, we have

$$
(\lambda-A)^{-1} f=\int_{0}^{\infty} e^{-(\lambda-s) t} f(t) d t
$$

belongs in $E$. Then $\rho(A)=\{\lambda \in \mathbb{C}: \Re \lambda>s\}$. Therefore, we obtain that the conclusion of Theorem 2.7 does not hold. The same conclusion can be made in the ultradistribution case. Consider the spaces

$$
\begin{aligned}
& E_{(h)}=\left\{f \in C^{\infty}(\mathbb{R}): f(0)=0, p_{n}(f)=\sup _{k \in \mathbb{N}_{0}} \sup _{t \geq k} \frac{h^{n}\left|f^{(n)}(t)\right|}{M_{n}}<+\infty\right\} \\
& E_{\{h\}}=\left\{f \in C^{\infty}(\mathbb{R}): f(0)=0, p_{n}(f)=\sup _{k \in \mathbb{N}_{0}} \sup _{t \geq k} \frac{h^{n}\left|f^{(n)}(t)\right|}{M_{n} \prod_{i=1}^{n} r_{i}}<+\infty\right\}
\end{aligned}
$$

for $\left(r_{i}\right)$ monotonically increasing positive sequence and $\left(M_{n}\right)$ satisfying $(M .1)$ and $(M .3)^{\prime}$. The spaces $E_{(h)}$ and $E_{\{h\}}$ are Fréchet spaces. Let $A=-\frac{d}{d s}$ with domain $D(A)=\left\{f \in E_{h}: f(0)=0, A f \in E_{h}\right\}$, where $E_{h}$ stands for both spaces. Let $p_{n} \in \circledast$ be a seminorm in $E_{\{h\}}$. The previous consideration in distribution case for $E=\mathcal{S}(\mathbb{R})$ is similar and more simple then the case with spaces $E_{(h)}$ and $E_{\{h\}}$.

Theorem 2.9. Let $A$ be a stationary dense operator in a sequentially complete locally convex space $E, n=n(A)$ and $F=\overline{D\left(A^{n}\right)}$. Then $A$ generates a distribution semigroup in $E$ if and only if $A_{F}$ generates a distribution semigroup in $F$.

Proof. The proof of this theorem is direct consequence of Theorem 1.2 Lemma 2.4 and Lemma 2.6 b).

Following two results are due to T. Ushijima and we can restate it in locally convex case as following.

Theorem 2.10. Let $E$ be a sequentially complete locally convex space and $A$ be a closed operator with dense $D\left(A^{\infty}\right)$. Then $A$ has the property $(E Q)$ if and only if there exists logarithmic region $\Omega_{\alpha, \beta}$ and $k \in \mathbb{N}$ and $C>0$ such that

$$
\|R(\lambda: A)\| \leq C(1+|\lambda|)^{k}, \quad \lambda \in \Omega_{\alpha, \beta} .
$$

Theorem 2.11. Let $E$ be a sequentially complete locally convex space and $A$ linear operator on $E$. The following conditions are equivalent:

i) $A$ is the generator of a distribution semigroup $G$;

ii) $A$ is well-posed and densely defined;

iii) A satisfy the condition (EQ);

iv) $A$ is densely defined, and there exist an adjoint logarithmic region $\Omega_{\alpha, \beta}$ and $k \in \mathbb{N}$ and $C>0$ such that

$$
\|R(\lambda: A)\| \leq C(1+|\lambda|)^{k}, \quad \lambda \in \Omega_{\alpha, \beta} .
$$

Proof. We will prove $i) \Rightarrow i i) \Rightarrow i i i) \Rightarrow i v) \Rightarrow i$ ). The statements $i) \Leftrightarrow i i$ ) and $i v) \Rightarrow i$ ) follow from Theorem 1.2 and $i i i) \Rightarrow i v$ ) follows by Theorem 2.10 It remains to show that $i) \Rightarrow i i i)$ and $i v) \Rightarrow i i)$.

$i) \Rightarrow$ iii $)$ : By the definition of the distribution semigroup, we can conclude that 
$D\left(A^{\infty}\right)$ contains $\mathcal{R}(G)$. Since $\mathcal{R}(G)$ is dense in $E, D\left(A^{\infty}\right)$ is dense in $E$. By the results in the third section from [19], we obtain that

$$
\left(\boldsymbol{\lambda}-\mathbf{A}_{\infty}\right)^{-1}(1 \otimes x)(\hat{\varphi})=G(\varphi) x,
$$

for any $\varphi \in \mathcal{D}$ and $x \in D_{\infty}(A)$. Note that with $\left(\boldsymbol{\lambda}-\mathbf{A}_{\infty}\right)^{-1}$ is denoted the generalized resolvent. Let $f_{\lambda}(t)=\mu(t) e^{-\lambda t}$, where $\mu \in \mathcal{D}$ and $\mu(t)=1$ for $|t| \leq 1$. Define the operator $R_{\infty}(\lambda)=G\left(f_{\lambda}\right)$. Since $G \in \mathcal{D}_{0}^{\prime}(L(E))$, for all $p \in \circledast$, there exists $q \in \circledast, k \in \mathbb{N}, C>0$ such that for all $x \in E, \lambda \in \mathbb{C}, \Re \lambda \geq 0$

$$
p\left(R_{\infty}(\lambda) x\right) \leq C(1+|\lambda|)^{k} q(x) .
$$

Now, for any $x \in D_{\infty}(A), \varphi \in \mathcal{D}, a>0$,

$$
\begin{gathered}
\frac{1}{i} \int_{a-i \infty}^{a+i \infty} \hat{\varphi}(\lambda) R_{\infty}(\lambda) x d \lambda=\int_{a-i \infty}^{a+i \infty}\left(\frac{1}{2 \pi i} \int_{-\infty}^{\infty} \varphi(t) e^{t \lambda} d t\right) G\left(f_{\lambda}\right) d \lambda x= \\
=G\left(\mu(s) \frac{1}{2 \pi i} \int_{a-i \infty}^{a+i \infty} d \lambda \int_{-\infty}^{+\infty} \varphi(t) e^{(t-s) \lambda} d t\right) x=G(\mu \cdot \varphi) x= \\
=G(\varphi) x=\left(\boldsymbol{\lambda}-\mathbf{A}_{\infty}\right)^{-1}(1 \otimes x)(\hat{\varphi}) .
\end{gathered}
$$

Again, using third section in [19], we obtain

$$
\int_{0}^{\infty} \varphi(t) T_{\infty}(t) x d t=G(\varphi) x
$$

for all $\varphi \mathcal{D}$ and $x \in D_{\infty}(A)$, which gives $\left.\left.\left.i i i\right) . v\right) \Rightarrow i i\right)$ : It is same like in $(v) \Rightarrow(i i)$ of [18, Theorem 4.1].

Theorem 2.12. Let $A$ be a closed operator in $E$. Then $A$ generates a distribution semigroup in $E$ if and only if $A$ is stationary dense and $A_{\infty}$ generates an equicontinuous semigroup in $D_{\infty}(A)$.

Proof. Let $A$ generates distribution semigroup in $E$. By simpler version of Theorem 1.2 and Lemma 2.4. $A$ is stationary dense. Now, let $n=n(A)$ and $F=\overline{D\left(A^{n}\right)}$. Then by Lemma 2.6 a) and Theorem 2.9 $A_{F}$ generates a dense distribution semigroup in $F$. Then $\left(A_{F}\right)_{\infty}$ generates an equicontinuous semigroup in $D_{\infty}\left(A_{F}\right)$. By Lemma 2.6 c) follows the conclusion.

Opposite direction. Let $A$ is stationary dense and $A_{\infty}$ generates an equicontinous semigroup and $n=n(A)$ and $F=\overline{D\left(A^{n}\right)}$. Then $A_{F}$ generates a distribution semigroup in $F$. By Theorem 2.9 we obtain that $A$ generates a distribution semigroup in $E$.

Theorem 2.13. Let $A$ be a closed operator in E. Then $A$ generates an exponential distribution semigroup in $E$ if and only if $A$ is stationary dense and $A_{\infty}$ generates a quasi-equicontinuous semigroup in $D_{\infty}(A)$.

The proof is direct consequence by the results of exponential distribution semigroups listed before. 


\section{REFERENCES}

[1] Da Prato G., Sinestrari E., Differential operators with nondense domain, Ann. Scuola Norm. Sup. Pisa Cl. Sci. (4) 14 (1987), 285-344.

[2] Fujiwara D., A characterisation of exponential distribution semigroups, J. Math. Soc. Japan 18 (1966), 267-275.

[3] Komatsu H., Semi-groups of operators in locally convex spaces, J. Math. Soc. Japan 16 (1964), 230-262.

[4] Kōmura T., Semigroups of operators in locally convex spaces, J. Funct. Anal. 2 (1968), $258-296$.

[5] Kostić M., Generalized Semigroups and Cosine Functions, Mathematical Institute Belgrade, 2011.

[6] Kostić M., Abstract Volterra Integro-Differential Equations, Taylor and Francis Group/CRC Press, 2015.

[7] Kostić M., C-Distribution semigroups, Studia Math. 185 (2008), 201-217.

[8] Kostić M., Pilipović S., Ultradistributions of semigroups, Sibirsk. Mat. Zh. 53 (2012), 291-305 (in Russian); Siberian Math. J. 53 (2012), 232-242.

[9] Kostić M., Pilipović S., Velinov D., Structural theorems for ultradistribution semigroups, Siberian Math J. 561 (2015), 83-91.

[10] Kostić M., Pilipović S., Velinov D., Hyperfunction semigroups, Siberian Math. J. 564 (2015), $650-661$.

[11] Kunstmann P. C., Stationary dense operators and generation of non-dense distribution semigroups, J. Operator Theory 37 (1997), 111-120.

[12] Kunstmann P. C., Distribution semigroups and abstract Cauchy problems, Trans. Am. Math. Soc. 351 (1999), 837-856.

[13] Kunstmann P. C., Banach space valued ultradistributions and applications to abstract Cauchy problems, preprint.

[14] Kunstmann P. C., Mijatović M., Pilipović S., Classes of distribution semigroups, Studia Math. 187 (2008), 37-58.

[15] Lions J. L., Semi-groupes distributions, Port. Math. 19 (1960), 141-164.

[16] Schwartz L., Théorie des distributions à valeurs vectorielles. I, Annales de l'institut Fourier 7 (1957) $1-141$.

[17] Melnikova I. V., Filinkov A.I., Abstract Cauchy Problems: Three Approaches, Chapman Hall/CRC, 2001.

[18] Ushijima T., On the generation and smoothness of semi-groups of linear operators, J. Fac. Sci., Univ. Tokyo, Sect. I A 19 (1972), 65-126.

[19] Ushijima T., On the abstract Cauchy problem and semi-groups of linear operators in locally convex spaces, Sci. Pap. Coll. Gen. Educ., Univ. Tokyo 21 (1971), 93-122.

Department for Mathematics, Faculty of Civil Engineering, Ss. Cyril and Methodius

University, Skopje, Partizanski Odredi 24, P.O. Box 560, 1000 Skopje

E-mail address: velinovd@gf.ukim.edu.mk

Faculty of Technical Sciences, University of Novi Sad, Trg D. Obradovića 6, 21125

Novi SAD, SERBia

E-mail address: marco.s@verat.net

Department for Mathematics and Informatics, University of Novi Sad, Trg D. Obradovića

4, 21000 Novi Sad, Serbia

E-mail address: pilipovic@dmi.uns.ac.rs 